\title{
Strong cosmic censorship for a scalar field in an Einstein-Maxwell-Gauss-Bonnet-de Sitter black hole
}

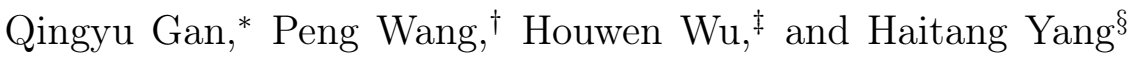 \\ Center for Theoretical Physics, College of Physics, \\ Sichuan University, Chengdu, 610064, PR China
}

\begin{abstract}
It has been shown that the Christodoulou version of the Strong Cosmic Censorship (SCC) conjecture can be violated for a scalar field in a near-extremal Reissner-Nordstrom-de Sitter black hole. In this paper, we investigate the effects of higher derivative corrections to the Einstein-Hilbert action on the validity of SCC, by considering a neutral massless scalar perturbation in 5- and 6dimensional Einstein-Maxwell-Gauss-Bonnet-de Sitter black holes. Our numerical results exhibit that the higher derivative term plays a different role in the $d=5$ case than it does in the $d=6$ case. For $d=5$, the SCC violation region increases as the strength of the higher derivative term increases. For $d=6$, the SCC violation region first increases and then decreases as the higher derivative correction becomes stronger, and SCC can always be restored for a black hole with a fixed charge ratio when the higher derivative correction is strong enough. Finally, we find that the $C^{2}$ version of SCC is respected in the $d=6$ case, but can be violated in some near-extremal regime in the $d=5$ case.
\end{abstract}

\footnotetext{
*Electronic address: gqy@stu.scu.edu.cn

${ }^{\dagger}$ Electronic address: pengw@scu.edu.cn

‡Electronic address: iverwu@scu.edu.cn

$\S$ Electronic address: hyanga@scu.edu.cn
} 


\section{Contents}

I. Introduction

II. Einstein-Maxwell-Gauss-Bonnet-de Sitter Black Hole 5

III. Quasinormal Mode $\quad 7$

IV. Numerical Results

A. $\mathbf{d}=\mathbf{5}$

B. $\mathbf{d}=\mathbf{6}$

V. Discussion and Conclusion

References 18

\section{INTRODUCTION}

It is well known that a curvature singularity could be formed during a gravitational collapse. There might exist three types of the singularities, namely space-like, light-like and time-like ones. Among them, the undetermined initial data on a time-like singularity would cause the breakdown of determinism of general relativity. Although there exist some solutions to the Einstein's equation admitting time-like singularities (e.g., Kerr-Newman and Reissner-Nordstrom black hole solutions), claiming that general relativity could lose predictability is rather subtle due to the presence of the Cauchy horizon, which encloses the time-like singularity. In particular, to rescue the predictability of general relativity, Penrose proposed the Strong Cosmic Censorship (SCC) conjecture, which asserts that the maximal Cauchy development of physically acceptable initial conditions is locally inextendible as a regular manifold [1-3]. Consequently, when the initial data is perturbed outside of a black hole, whether SCC holds true crucially depends on the extensibility of the perturbation (e.g., the metric and other fields) at the Cauchy horizon.

To give a more rigorous definition of the extensibility of the perturbation across the Cauchy horizon, several formulation versions of SCC have been proposed. For example, the $C^{r}$ version of SCC states that the metric can not be $C^{r}(r \in N)$ smooth at the Cauchy 
horizon [4, 5]. Various evidences suggest that the Cauchy horizon can be extendible with a continuous metric for the perturbed initial data, indicating the falsity of the $C^{0}$ version of SCC [6-8]. On the other hand, it has been argued that the $C^{2}$ version of SCC appears to be true since the curvature blows up at the Cauchy horizon [9]. However, an observer can still experience a finite tidal force and cross the Cauchy horizon even when the metric is inextendible in $C^{2}$ [10]. So requiring that the metric is $C^{2}$ at the Cauchy horizon seems to be too strong, and extensions with lower smoothness shall be considered.

It is worth noting that weak solutions can have many important physical applications, in which $C^{r}$ smooth solutions are not available. Therefore it might be a more appropriate choice to characterize the extensibility of the Cauchy horizon by considering whether the perturbation is inextendible as a weak solution. For the Einstein's equation, a weak solution is specified by locally square integrable Christoffel symbols in some charts of the manifold. This observation then leads to the Christodoulou formulation of SCC, which states that the maximal Cauchy development should be inextendible as a spacetime with locally square integrable Christoffel symbols [11]. Practically, for a linear scalar perturbation, the Christodoulou version of SCC can be tested by checking whether the scalar field will belong to the Sobolev space $H_{l o c}^{1}$ at the Cauchy horizon. Note that if the perturbation belongs to the Sobolev space $H_{l o c}^{1}$, its first derivative is locally square integrable. In other words, if SCC is violated in the Christodoulou version, the perturbation belongs to $H_{l o c}^{1}$ and, roughly speaking, has finite energy at the Cauchy horizon.

To check the validity of the SCC, one needs to analyze the evolution of the perturbation, which is governed by two mechanisms. One is the mass-inflation mechanism, associated with the exponential amplification of a perturbation due to the blue shift effect, which might cause a singular behavior at the Cauchy horizon [7, 9, 10, 12, 14. The other is the time-dependent remnant perturbation decaying outside of the black hole, which can compete with the mass inflation to invalidate SCC. For an asymptotically flat black hole with the perturbation outside the black hole decaying in an inverse power-law way, the mass-inflation mechanism dominates to render the Cauchy horizon unstable [7, 15 18]. Interestingly, the exponentially decay of the perturbation is observed in asymptotically dS spacetime, indicating that mass inflation might not be strong enough to keep SCC valid. Quantitatively, for a linear scalar perturbation in an asymptotic dS black hole, the competition between the the mass inflation 
and remnant decaying is characterized by [19-24]

$$
\beta \equiv \frac{\alpha}{\kappa_{-}}
$$

where $\kappa_{-}$denotes the surface gravity at the Cauchy horizon, and $\alpha$ is the spectral gap representing the distance from real axis to the lowest-lying QuasiNormal Mode (QNM) on the lower half complex plane of frequency. It showed that $\beta>1 / 2$ corresponds to the violation of the Christodoulou version of SCC. Moreover, $\beta>1$ represents the $C^{1}$ extensibility of a scalar field at the Cauchy horizon, leading to the bounded curvature if coupled to gravity [19, 23, 25]. Hence $\beta>1$ implies the violation of SCC in the $C^{2}$ version, opening the possibility to the existence of solutions with even higher regularity across the Cauchy horizon. From now on, the term "SCC" only refers to the Christodoulou version of SCC.

Recently, the validity of SCC has been widely explored in asymptotic dS black holes. In particular, the authors in [23, 26, 31] considered scalar perturbations with/without mass and charge in a Reissner-Nordstrom-de Sitter (RNdS) black hole, and found that SCC is violated in the near-extremal region. The analysis has been extended to the Dirac field perturbation [32 35] and higher space-time dimensions [36 38], where there still exists some room for the violation of SCC. Especially in [26, 33], it has been observed that the $C^{2}$ version of SCC can be violated since $\beta>1$ appears in some near-extremal parameter regimes. Even worse, if one considers the case with the coupled linearized electromagnetic and gravitational perturbations in a RNdS black hole, the $C^{r}$ version of SCC for any $r \geq 2$ can be violated by taking the black hole close enough to extremality [25]. Moreover, the authors of [34, 39, 40] argued that nonlinear effects could not save SCC from being violated for a near-extremal RNdS black hole. Surprisingly, SCC can always be respected for the massless scalar field and linearized gravitational perturbations in a Kerr-dS black hole [36, 41].

It is interesting and inspiring to check the validity of SCC in models beyond the EinsteinMaxwell theory. In [42, 43], we studied SCC for dS black holes in the Einstein-Born-Infeld and Einstein-Logarithmic systems and found that the nonlinear electrodynamics effects tend to rescue SCC. In addition, SCC has been tested for a scalar field perturbation in the Horndeski theory in [44], which showed that the higher-order derivative couplings increases the regularity requirements for the existence of weak solutions beyond the Cauchy horizon. On the other hand, Gauss-Bonnet (GB) gravity, which arises from the low energy effective ac- 
tion of the heterotic string theory [45], has attracted considerable attention in the literature. Coupling to the Maxwell electrodynamics, namely in the Einstein-Maxwell-Gauss-Bonnet (EMGB) theory, the EMGB black hole solution was obtained, and various aspects have been extensively investigated [46 53]. To the best of our knowledge, little is known about the validity of SCC in the EMGB theory. The purpose of this paper is to investigate the validity of SCC for a neutral massless scalar perturbation propagating in 5- and 6-dimensional EMGBdS black holes.

The rest of the paper is organized as follows. In Sec.II, we briefly review the 5- and 6dimensional EMGBdS black hole solutions and obtain the allowed parameter regions, where the Cauchy horizon exists. In Sec.III, we show how to compute the QNMs for a neutral massless scalar perturbation in an EMGBdS black hole. In Sec.IV, we present and discuss the numerical results in various parameter regions. We summarize our results and conclude with a brief discussion in the last section. For simplicity, we set $G=c=1$ in this paper.

\section{EINSTEIN-MAXWELL-GAUSS-BONNET-DE SITTER BLACK HOLE}

In this section, we briefly review the EMGBdS black hole solution and obtain the parameter region where three horizons exists. The action of the Einstein-Maxwell-Gauss-Bonnet theory in $d$-dimensional spacetime is given by [46]

$$
S=\frac{1}{16 \pi} \int d^{d} x \sqrt{-g}\left[\mathcal{R}-2 \Lambda+\alpha_{G B}\left(R^{2}-4 R_{\mu \nu} R^{\mu \nu}+R_{\mu \nu \rho \sigma} R^{\mu \nu \rho \sigma}\right)-F^{\mu \nu} F_{\mu \nu}\right],
$$

where $\Lambda>0$ is the cosmology constant, $\mathcal{R}$ is the Ricci scalar curvature, and $F_{\mu \nu}=\partial_{\mu} A_{\nu}-$ $\partial_{\nu} A_{\mu}$ is the electromagnetic tensor field of the electromagnetic field $A_{\mu}$. It is noteworthy that the GB coupling constant $\alpha_{G B}$ is naturally assumed to be positive since the GB correction to the Einstein gravity is well motivated from the low energy effective action of the heterotic string theory 45]. So we focus on $\alpha_{G B} \geq 0$ in this paper. In addition, the GB term is known to be topological with no dynamics in $d=4$ dimension. Therefore, we shall consider $d \geq 5$ in what follows.

For the action (II.2), a static spherically symmetric black hole solution was obtained in [46, 48, 51]:

$$
d s^{2}=-f(r) d t^{2}+\frac{d r^{2}}{f(r)}+r^{2} d \Omega_{d-2}^{2}, \quad \mathbf{A}=A_{t} d t=-\frac{4 \pi Q}{(d-3) \omega_{d-2} r^{d-3}} d t
$$


with the blackening factor

$f(r)=1+\frac{r^{2}}{2 \widetilde{\alpha}}-\frac{r^{2}}{2 \widetilde{\alpha}} \sqrt{1+4 \widetilde{\alpha}\left[\frac{2 \Lambda}{(d-2)(d-1)}+\frac{16 \pi M}{(d-2) \omega_{d-2} r^{d-1}}-\frac{32 \pi^{2} Q^{2}}{(d-2)(d-3) \omega_{d-2}^{2} r^{2 d-4}}\right]}$,

where $M$ and $Q$ are the ADM mass and the electric charge of the EMGBdS black hole, respectively, and $d \Omega_{d-2}^{2}$ represents the line element of a $(d-2)$-dimensional unit sphere with volume $\omega_{d-2}=2 \pi^{(d-1) / 2} / \Gamma((d-1) / 2)$. For simplify, we introduce a redefined GB parameter $\widetilde{\alpha}=\alpha_{G B}(d-3)(d-4)$. In the limit of $\widetilde{\alpha} \rightarrow 0$, eqns. (II.3) and (II.4) reduce to the $d$-dimensional RNdS black hole as expected [37].

An EMGBdS black hole is characterized by the parameters $M, Q, \Lambda$ and $\widetilde{\alpha}$. It can show that the EMGBdS black hole can possess one, two or three horizons in different parameter regimes. The topology and causal structure of EMGBdS black holes have been detailedly analyzed in [50]. To study SCC, we need to find the "allowed" region in parameter space, in which the black hole possesses three horizons, namely the Cauchy horizon $r_{-}$, the event horizon $r_{+}$and the cosmological horizon $r_{c}$. For later use, we denote the surface gravity $\kappa_{h} \equiv\left|f^{\prime}\left(r_{h}\right)\right| / 2$ with $h \in\{+,-, c\}$ for each horizon. The allowed region is determined by the two limits, namely the extremal limit with $r_{-}=r_{+}$, which corresponds to the extremal black hole with charge $Q_{\text {ext }}$, and the Nariai limit with $r_{+}=r_{c}$, which corresponds to the Nariai black hole with charge $Q_{\text {nar }}$. Hence, the allowed region is given by $Q_{\text {nar }}<Q<Q_{\text {ext }}$ or $0<Q<Q_{\text {ext }}$ if no Nariai limit exists. In particular, a 5-dimensional EMGBdS black hole has $Q_{\text {nar }}=\pi \sqrt{-2+3 k-2(1-k)^{3 / 2}} / \Lambda$ and $Q_{\text {ext }}=\pi \sqrt{-2+3 k+2(1-k)^{3 / 2}} / \Lambda$ with $k \equiv \Lambda(8 M-3 \pi \widetilde{\alpha}) /(6 \pi)$ [54]. The existence of $Q_{\text {nar }}$ and $Q_{\text {ext }}$ requires $\widetilde{\alpha}<8 M /(3 \pi)$, which puts an upper bound on $\alpha$. If $\Lambda>\Lambda_{\mathrm{c}} \equiv 3 \pi /(4 M), Q_{\text {nar }}$ could only exist when $\widetilde{\alpha}>8 M /(3 \pi)-2 / \Lambda$, which puts a nonzero lower bound on $\alpha$. However for $d \geq 6$, the allowed region can only be numerically determined. For example, our numerical results show that, for $d=6$, there is no upper bound on $\widetilde{\alpha}$, and a nonzero lower bound on $\widetilde{\alpha}$ appears when $\Lambda>\Lambda_{\mathrm{c}} \sim 6.348 M^{-2 / 3}$.

The allowed regions and their boundaries in the $\widetilde{\alpha}-Q$ parameter space are plotted in Fig. 1 for various values of $\Lambda$ in the $d=5$ and $d=6$ cases. Without loss of generality, we set $M=1$ in the rest of this paper. When $\Lambda>\Lambda_{\mathrm{c}}$, the right column of Fig. 1 shows that the allowed regions have a nonzero minimum value of $\widetilde{\alpha}$, marked by the point $A$, as expected. One can also notice that, for the allowed region, $\widetilde{\alpha}<8 /(3 \pi)$ in the $d=5$ case while no 

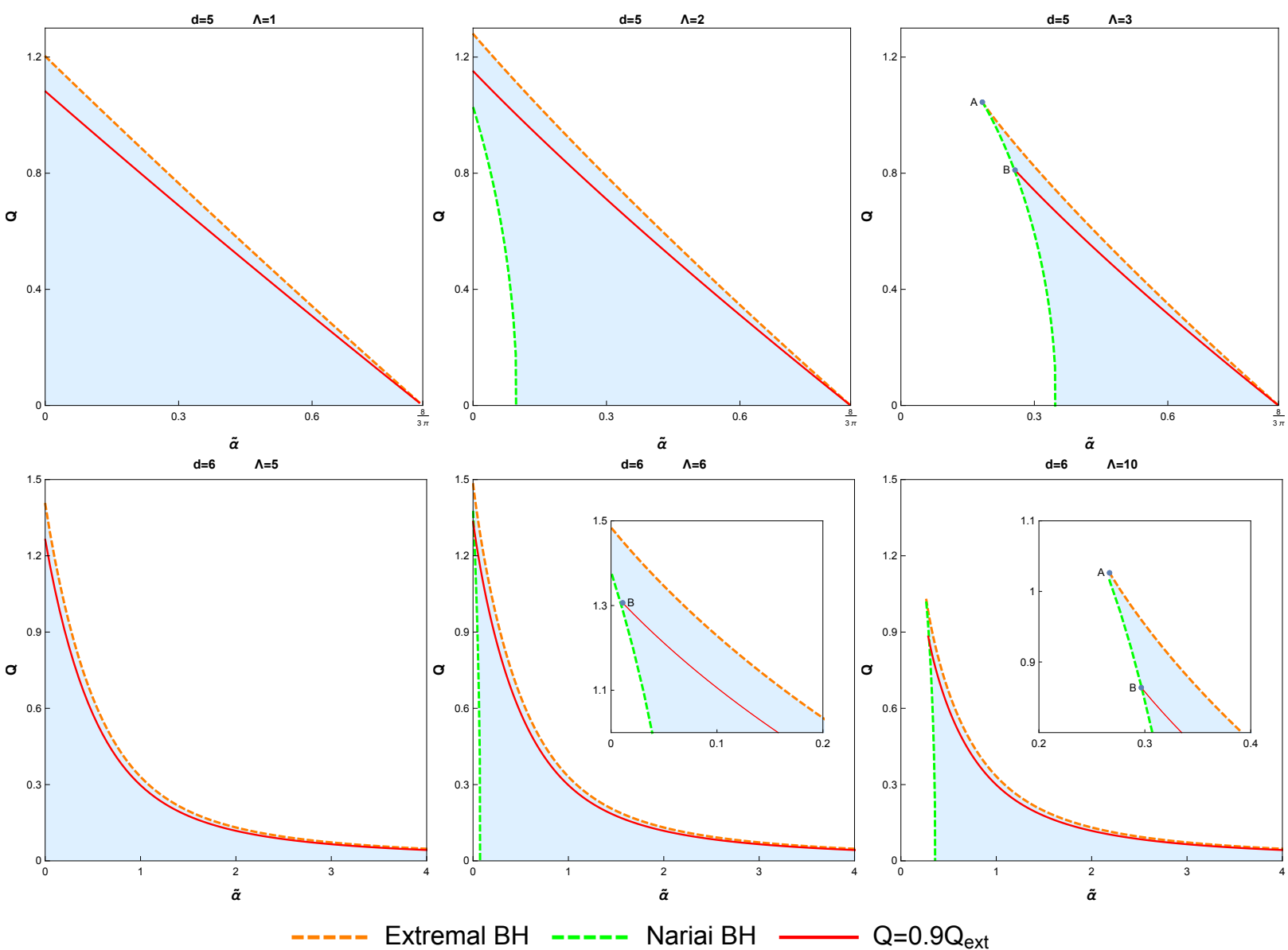

FIG. 1: The regions in light blue are allowed to possess three horizons for EMGBdS black holes in $d=5$ (upper row) and $d=6$ (lower row) for various values of $\Lambda$. The dashed orange and green lines represent the extremal black hole and the Nariai black hole, respectively. When $\Lambda>\Lambda_{\mathrm{c}}(\mathbf{r i g h t}$ column), there exists a tip point $A$, which marks a nonzero minimum value of $\widetilde{\alpha}$. The solid red line, which represents the near-extremal black hole with the charge ratio $Q / Q_{\text {ext }}=0.9$, intersects with the dashed green line at point $B$, where SCC tends to be saved as discussed below.

upper bound on $\widetilde{\alpha}$ exists in the $d=6$ case. Furthermore, we find that the allowed regions are in much similarity in the $d \geq 6$ cases. Thus we shall consider only two cases with $d=5$ and $d=6$ in what follows.

\section{QUASINORMAL MODE}

In this section, we discuss QNMs for a neutral massless scalar perturbation in the $d$ dimensional EMGBdS spacetime. The behavior of the neutral massless scalar field is gov- 
erned by the Klein-Gordon equation

$$
\nabla^{2} \Phi=0
$$

where $\nabla$ is the covariant derivative. To facilitate our numerical calculation, we use the Eddington-Finkelstein ingoing coordinates $\left(v, r, \boldsymbol{\Omega}_{d-2}\right)$ with $v=t+r_{*}$, where $r_{*}$ is the tortoise coordinate defined as $d r_{*}=d r / f(r)$. In addition, we choose an appropriate gauge transformation such that $\mathbf{A}=A_{v} d v=-4 \pi Q d v /\left((d-3) \omega_{d-2} r^{d-3}\right)$. Since the EMGBdS black hole solution is static and spherically symmetric, a mode solution of eqn. (III.5) can have the separable form

$$
\Phi\left(v, r, \boldsymbol{\Omega}_{d-2}\right)=\sum_{l m_{j}} \psi_{\omega l m_{j}}(r) Y_{l m_{j}}\left(\boldsymbol{\Omega}_{d-2}\right) e^{-i \omega v} .
$$

Here, $l$ and $m_{j}(j=1,2, \cdots, d-3)$ denote integers required to uniquely determine a $(d-2)$-hyperspherical harmonic $Y_{l m_{j}}\left(\boldsymbol{\Omega}_{d-2}\right)$, which fulfills $\nabla_{\mathbf{S}^{d-2}}^{2} Y_{l m_{j}}\left(\boldsymbol{\Omega}_{d-2}\right)=-l(l+d-$ 3) $Y_{l m_{j}}\left(\boldsymbol{\Omega}_{d-2}\right)$. Since no "magnetic splitting" is present due to the spherical symmetry of the background, the index $m_{j}$ can be suppressed in $\psi_{\omega l m_{j}}$ [55, 56]. Plugging eqn. (III.6) into eqn. (III.5), we obtain the radial equation

$$
\left(r^{2} f \partial_{r}^{2}+\left(-2 i \omega r^{2}+r^{2} f^{\prime}+(d-2) r f\right) \partial_{r}-i(d-2) \omega r-l(l+d-3)\right) \psi_{\omega l}(r)=0,
$$

where $f^{\prime}$ denotes $d f(r) / d r$.

One can perform the Frobenius method to obtain the solutions near the event and cosmological horizons, respectively. If we impose ingoing boundary condition at the event horizon and the outgoing boundary condition at the cosmological horizon, namely,

$$
\psi_{\omega l}^{\text {ingoing }}\left(r \rightarrow r_{h}\right) \sim \text { const., } \quad \psi_{\omega l}^{\text {outgoing }}\left(r \rightarrow r_{c}\right) \sim\left(r-r_{c}\right)^{-i \omega / \kappa_{c}},
$$

eqn. (III.7) selects a set of discrete frequencies $\omega_{l n}(n=1,2, \cdots)$, which are QNMs of the scalar field [57]. There are many analytic and numerical ways to extract QNMs [57, 58]. In this paper, we employ the Chebyshev collocation scheme and the associated Mathematica package developed in [59 61]. The basic idea to compute the spectrum efficiently is to discretize the QNM equations by the pseudospectral method and solve the resulting generalized eigenvalue equation. It can produce an additional infinite set of purely imaginary modes, which are known to be missed by the WKB approximation [57]. Moreover, WKB approximation assumes that the potential has a single extremum, which may fail in some cases [62]. 
To adapt to our numerical scheme in the Mathematica package, we redefine field $\psi_{\omega l}$ as

$$
\psi_{\omega l}=\frac{1}{x}(1-x)^{-i \omega / \kappa_{c}} \phi_{\omega l},
$$

with a new coordinate $x \equiv\left(r-r_{+}\right) /\left(r_{c}-r_{+}\right)$, which renders the new field $\phi_{\omega l}$ regular at both the event and cosmological horizons. After the radial equation for $\phi_{\omega l}$ is obtained from eqns. (III.7) and (III.9), one can use the package to find a series of QNMs, $\omega_{l n}$. The spectral gap $\alpha$ in eqn. II.1 is then given by $\alpha=\inf _{l n}\left\{-\operatorname{Im} \omega_{l n}\right\}$.

\section{NUMERICAL RESULTS}

In this section, we present the numerical results about QNMs for a neutral massless scalar perturbation in 5- and 6-dimensional EMGBdS black holes and check the validity of SCC. These results are obtained using the Mathematica package of [59 61] and found to be consistent with the results of [37, 49, 63, 64] in various limits. Since it showed that SCC could be violated in a near extremal RNdS black hole, we will focus on the near-extremal regime of the EMGBdS black holes.

\section{A. $\mathbf{d}=\mathbf{5}$}

We first study the $d=5$ case. For a near-extremal black hole, it is well known that there exist three qualitatively different families of QNMs: the photon sphere (PS) family, which can be traced back to the photon sphere, the de Sitter ( $d S$ ) family, which is deformation of the pure de Sitter modes, and the near-extremal (NE) family, which only appears for near-extremal black holes [23, 33, 37, 42, 65]. We plot these three distinct families for a 5-dimensional near-extremal EMGBdS black hole in Fig. 2, where we consider two cases with $\Lambda=1$ and $\Lambda=3$ since their allowed regions are quite different as depicted in Fig. 1 . As shown in Fig. 2a, when $Q / Q_{\text {ext }}$ increases toward the extremal limit, $\operatorname{Im}(\omega) / \kappa_{-}$for the PS and dS dominant modes become divergent while NE mode takes over to make $\beta$ finite but smaller than $-1 / 2$. Therefore, like a RNdS black hole, the presence of NE mode can invalidate SCC as long as the EMGBdS black hole lies close enough to extremality. As one increases $\widetilde{\alpha}$ from the left column to the right column in Fig. 2a, the SCC violation range of $Q / Q_{\text {ext }}$ expands, which implies that the GB term in the action (II.2) tends to worsen 


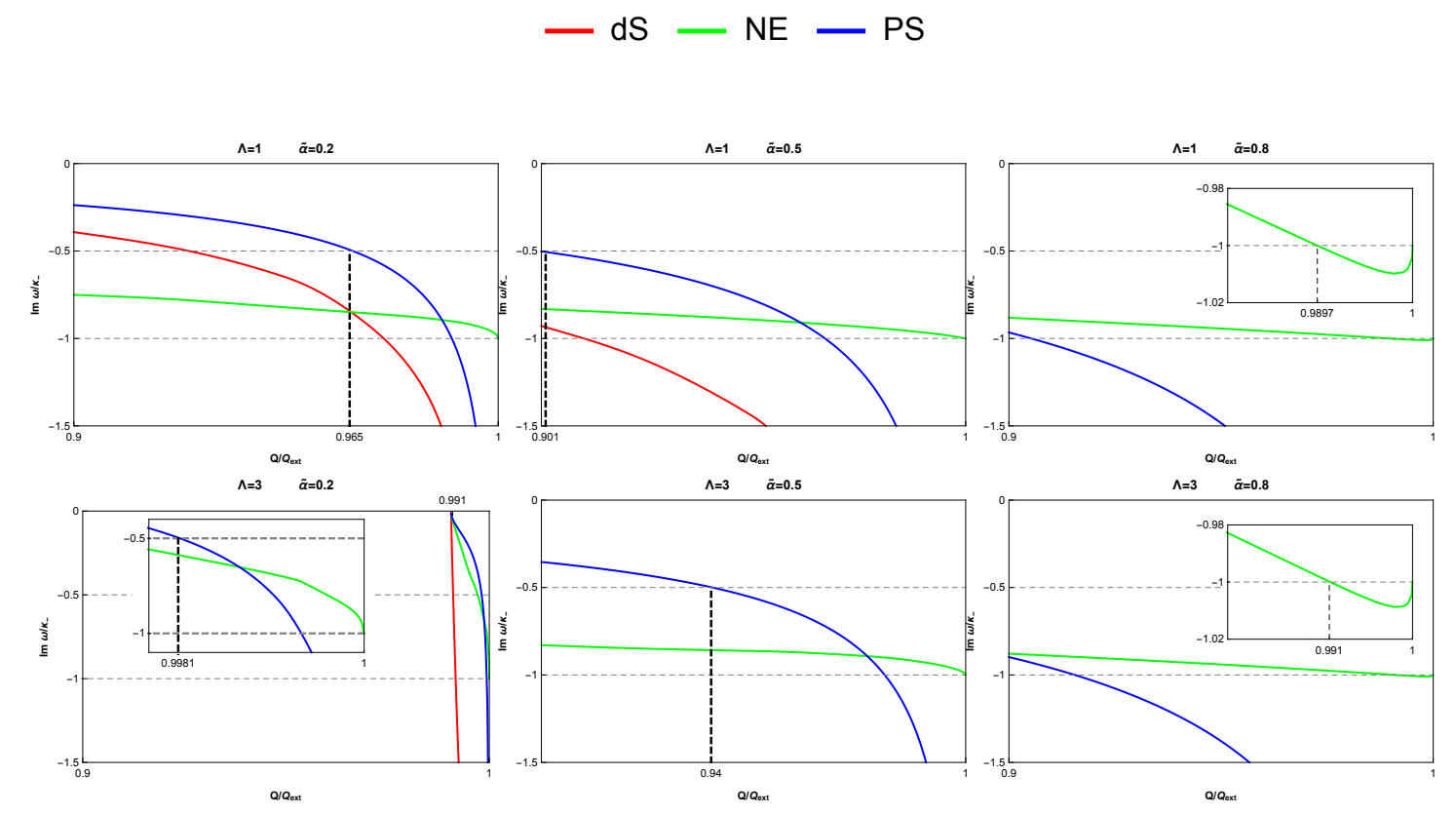

(a) Dominant modes of three families for various values of $\widetilde{\alpha}$ and $\Lambda$ with varying $Q / Q_{\text {ext }}$. The SCC violation range of $Q / Q_{\text {ext }}$ expands as $\widetilde{\alpha}$ increases.
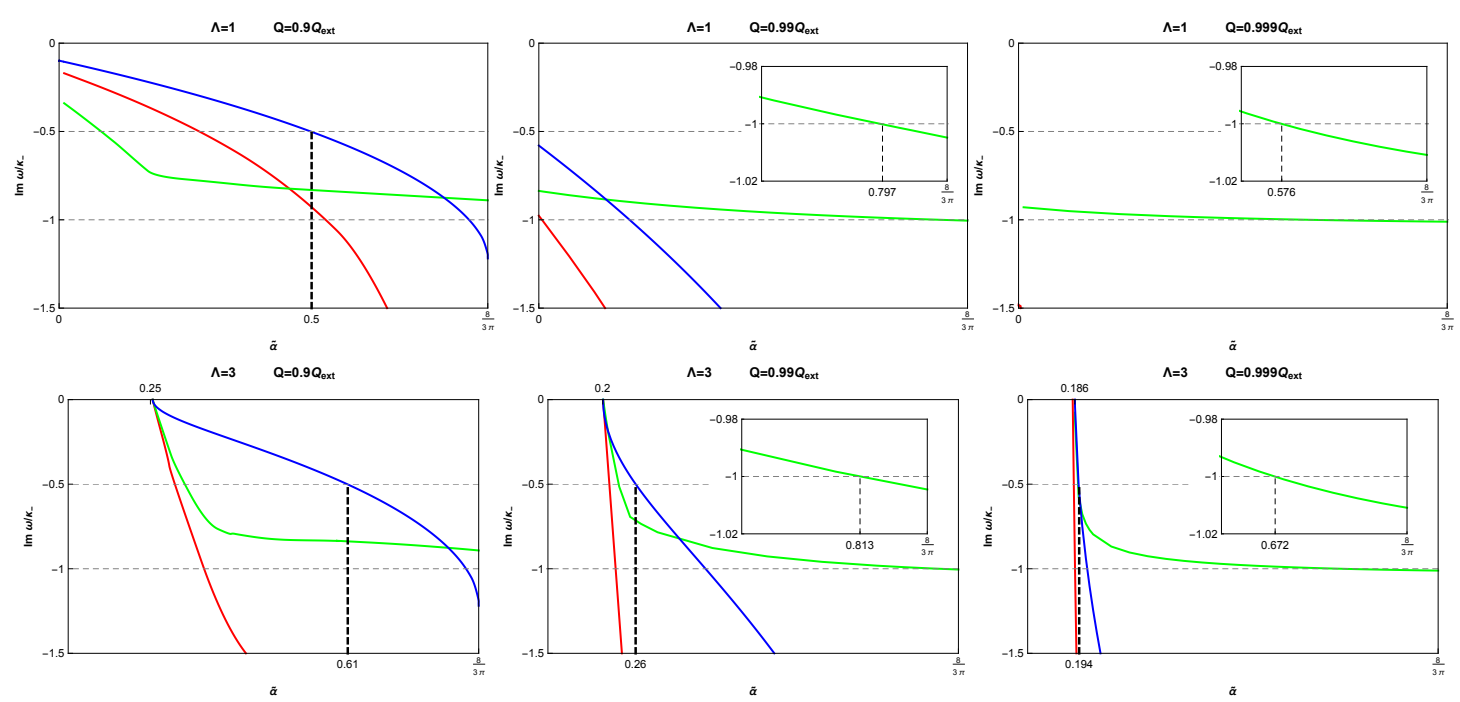

(b) Dominant modes of three families for various values of $Q / Q_{\text {ext }}$ and $\Lambda$ with varying $\widetilde{\alpha}$. SCC is violated when $\widetilde{\alpha}$ is large enough.

FIG. 2: Dominant modes of three families for a neutral massless scalar field in a 5-dimensional EMGBdS black hole, showing the dominant NE mode (green lines) at $l=0$, the dominant $\mathrm{dS}$ mode (red lines) at $l=1$ and the (nearly) dominant complex PS mode (blue lines) at $l=10$. The threshold $\beta \equiv-\operatorname{Im}(\omega) / \kappa_{-}=1 / 2$ is designated by thick dashed vertical lines, on the right of which $\mathrm{SCC}$ is violated. The dashed vertical lines in the insets denote $\beta=1$. 
the SCC violation for a scalar in a 5-dimensional near-extremal EMGBdS black hole. To better understand how the GB term affects the validity of SCC, we plot $\operatorname{Im}(\omega) / \kappa_{-}$against $\widetilde{\alpha}$ with increasing $Q / Q_{\text {ext }}$ from the left column to the right column in Fig. $2 \mathrm{~b}$. The SCC violation range of $\widetilde{\alpha}$, which are on the right of the thick dashed vertical lines, increases as $Q / Q_{\text {ext }}$ increases, indicating that SCC tends to be violated when the black hole is closer to extremality. Note that there is an upper bound $\widetilde{\alpha}=8 /(3 \pi)$ on $\widetilde{\alpha}$ for $d=5$ as discussed before. The $\operatorname{Im}(\omega) / \kappa_{-}$of all three families' dominant modes decrease to some finite values with $\widetilde{\alpha}$ increasing toward the upper bound. Fig. $2 \mathrm{~b}$ displays that, for a scalar in a 5dimensional near-extremal EMGBdS black hole, SCC is always violated when $\widetilde{\alpha}$ is close enough to the upper bound.

When $\Lambda=3>\Lambda_{\mathrm{c}}$, the upper right panel of Fig. 11 exhibits that there are a lower bound on $\widetilde{\alpha}$ with fixed $Q / Q_{\text {ext }}$ and a possible lower bound on $Q$ with fixed $\widetilde{\alpha}$. The black hole with the minimum value of $\widetilde{\alpha}$ or $Q$ (e.g., the point $B$ in Fig. 1) corresponds to the Nariai limit. The WKB method gives that the width and peak of the potential are small in the near Nariai regime, which makes QNMs vanished in the Nariai limit [23, 57, 58]. On the other hand, the surface gravity $\kappa_{-}$at the Cauchy horizon remains finite in the Nariai limit, which makes $\beta=0$ for the Nariai black hole. So it is expected to observe that $\beta=0$ at the minimum values of $Q / Q_{\text {ext }}$ and $\widetilde{\alpha}$ in the lower left panel of Fig. $2 \mathrm{a}$ and the lower row of Fig. 2b, respectively. Consequently, SCC is always valid around the Nariai limit. Therefore when $\Lambda>\Lambda_{\mathrm{c}}$, even for a 5-dimensional highly near-extremal EMGBdS black hole, SCC is always saved as long as $\widetilde{\alpha}$ is close enough to its minimum value.

We display the density plots of $\beta$ for 5 -dimensional EMGBdS black holes with $\Lambda=1$ and $\Lambda=3$ in Fig. 3. SCC is violated in the regions between the extremal lines (dashed orange lines) and the threshold $\beta=1 / 2$ (solid black lines). The $Q / Q_{\text {ext }}=0.9$ line in red shows that, in the both cases with $\Lambda=1$ and $\Lambda=3, \mathrm{SCC}$ is respected at small $\widetilde{\alpha}$ but violated when $\widetilde{\alpha}$ is large enough. For a more extremal black hole (e.g., $Q / Q_{\text {ext }}=0.99$ ), SCC could be always violated in the $\Lambda=1$ case. However when $\Lambda=3$, SCC can be recovered even for a highly near-extremal EMGBdS black hole in the region close to the Nariai line (dashed green lines).

Unlike the RNdS case [23], the insets in Fig. 2a show that there exist some near-extremal regions where the dominant $\mathrm{NE}$ mode dominates and has $\operatorname{Im}(\omega) / \kappa_{-}<-1$ (i.e., $\beta>1$ ), which indicates the violation of SCC in the $C^{2}$ version. Moreover, our numerical results in 

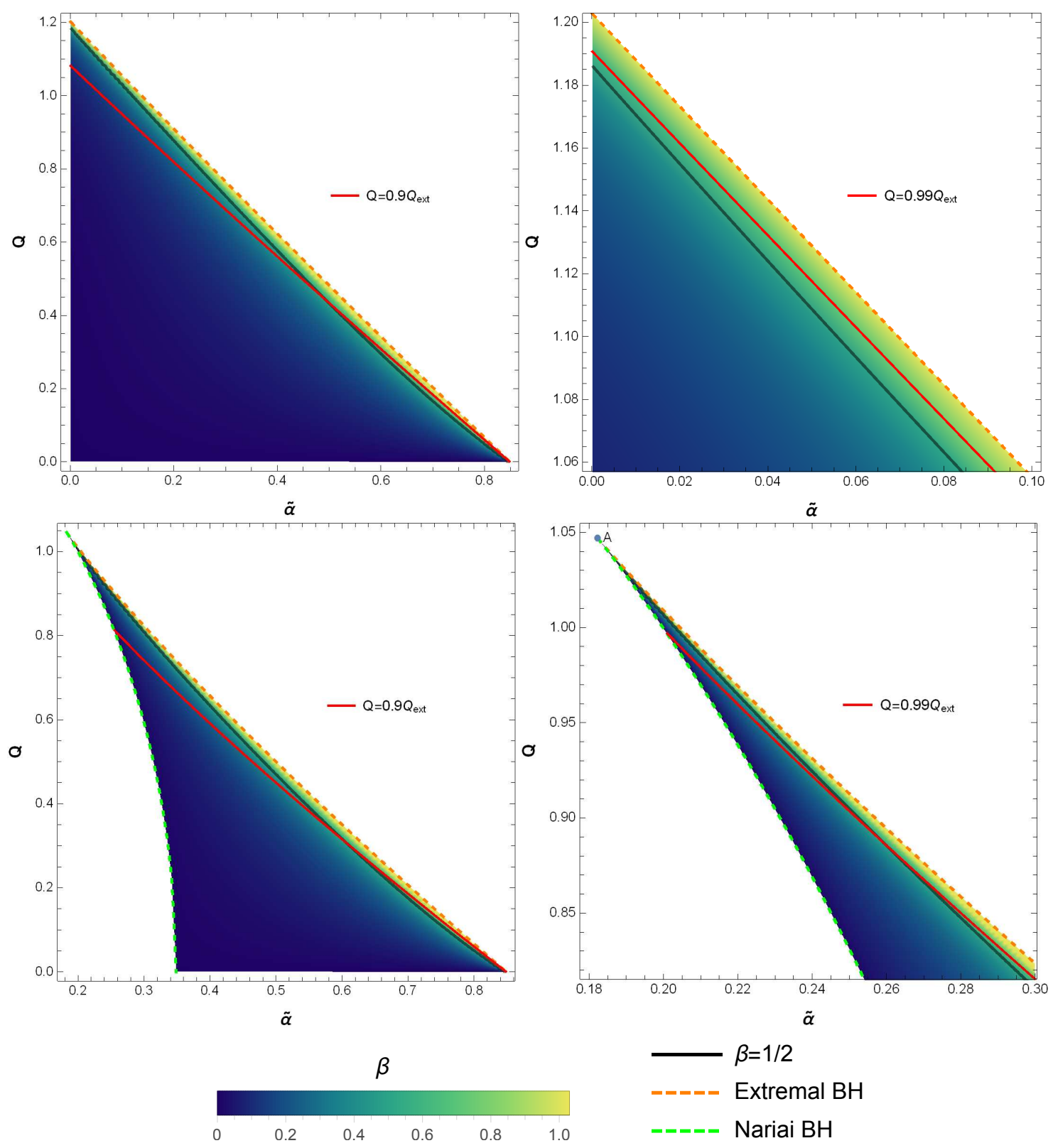

FIG. 3: Density plots of $\beta$ for a neutral massless scalar field in a 5-dimesinal EMGBdS black hole with $\Lambda=1$ (upper row) and $\Lambda=3$ (lower row). The parameter space of interest is bounded by the Nariai limit (dashed green lines) and the extremal limit (dashed orange lines). The solid black lines represent the threshold $\beta=1 / 2$. SCC is valid in the regions below the solid black lines. When $Q / Q_{\text {ext }}=0.9, \mathrm{SCC}$ is saved at small $\widetilde{\alpha}$ but violated at large $\widetilde{\alpha}$ for both $\Lambda=1$ and $\Lambda=3$. When $Q / Q_{\text {ext }}=0.99, \mathrm{SCC}$ is violated in all range of $\widetilde{\alpha}$ for $\Lambda=1$ but can be saved when $\widetilde{\alpha}$ is small enough for $\Lambda=3$. 


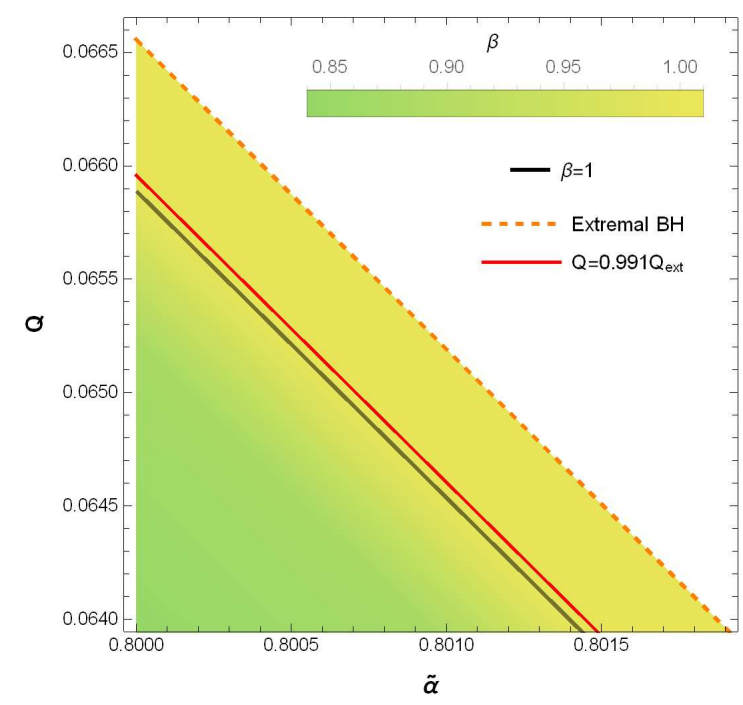

\begin{tabular}{|c|c|c|}
\hline$Q / Q_{e x t}$ & Im $\omega / K_{-}$ & $(N, p)$ \\
\hline $1-10^{-1}$ & $-0.881538(13)$ & $(200,50)(250,50)$ \\
\hline $1-10^{-2}$ & $-1.000480(11)$ & $(200,50)(250,50)$ \\
\hline $1-10^{-3}$ & $-1.009369(14)$ & $(450,50)(500,50)$ \\
\hline $1-10^{-4}$ & $-1.004804(7)$ & $(450,50)(500,50)$ \\
\hline $1-10^{-5}$ & $-1.0018(4)$ & $(600,50)(650,50)$ \\
\hline $1-10^{-6}$ & $-1.0006(3)$ & $(900,50)(950,50)$ \\
\hline
\end{tabular}

FIG. 4: Left Panel: Density plot of $\beta$ in a near-extremal region around $\widetilde{\alpha}=0.8$ with $\Lambda=1$ for $d=5$. Here the solid black line corresponds to the $\beta=1$. In the region between the solid black line and the extremal limit (dashed orange line), the $C^{2}$ version of SCC is violated for a near-extremal EMGBdS black hole, e.g., $Q / Q_{\text {ext }}=0.991$ shown in red line. Right Panel: A table of $\operatorname{Im}(\omega) / \kappa_{-}$of the dominant NE modes for various near-extremal values of $Q / Q_{\text {ext }}$ with $\Lambda=1$ and $\widetilde{\alpha}=0.8$. Numbers in brackets in the second column indicate the number of agreed digits after the decimal point. In the third column, we show the different grid size and precision $(N, p)$ used in the computations.

the table of Fig. 4 suggest that $\operatorname{Im}(\omega) / \kappa_{-}$for the dominant NE mode would approach -1 (i.e., $\beta \rightarrow 1$ ) in the extremal limit. In the density plot of $\beta$ displayed in Fig. $4, \beta>1$ and hence the $C^{2}$ version of SCC is violated in the region between the solid black line and the dashed orange line. Additionally, the insets in Fig. $2 \mathrm{~b}$ exhibit that when $Q / Q_{\text {ext }}=1-10^{-2}$ and $Q / Q_{\text {ext }}=1-10^{-3}$, the $C^{2}$ version of SCC can be violated for a large enough value of $\widetilde{\alpha}$, which means that GB term also tends to violate SCC in the $C^{2}$ version.

\section{B. $\quad d=6$}

We now consider QNMs for a scalar field in a 6-dimensional EMGBdS black hole and investigate the validity of SCC. Since the allowed regions in Fig. 1 are quite different for $\Lambda=5$ and $\Lambda=10$, we will focus on these two cases. For the lowest-lying mode of the three families, their $\operatorname{Im}(\omega) / \kappa_{-}$are depicted against $Q / Q_{\text {ext }}$ for various values of $\widetilde{\alpha}$ in Fig. 


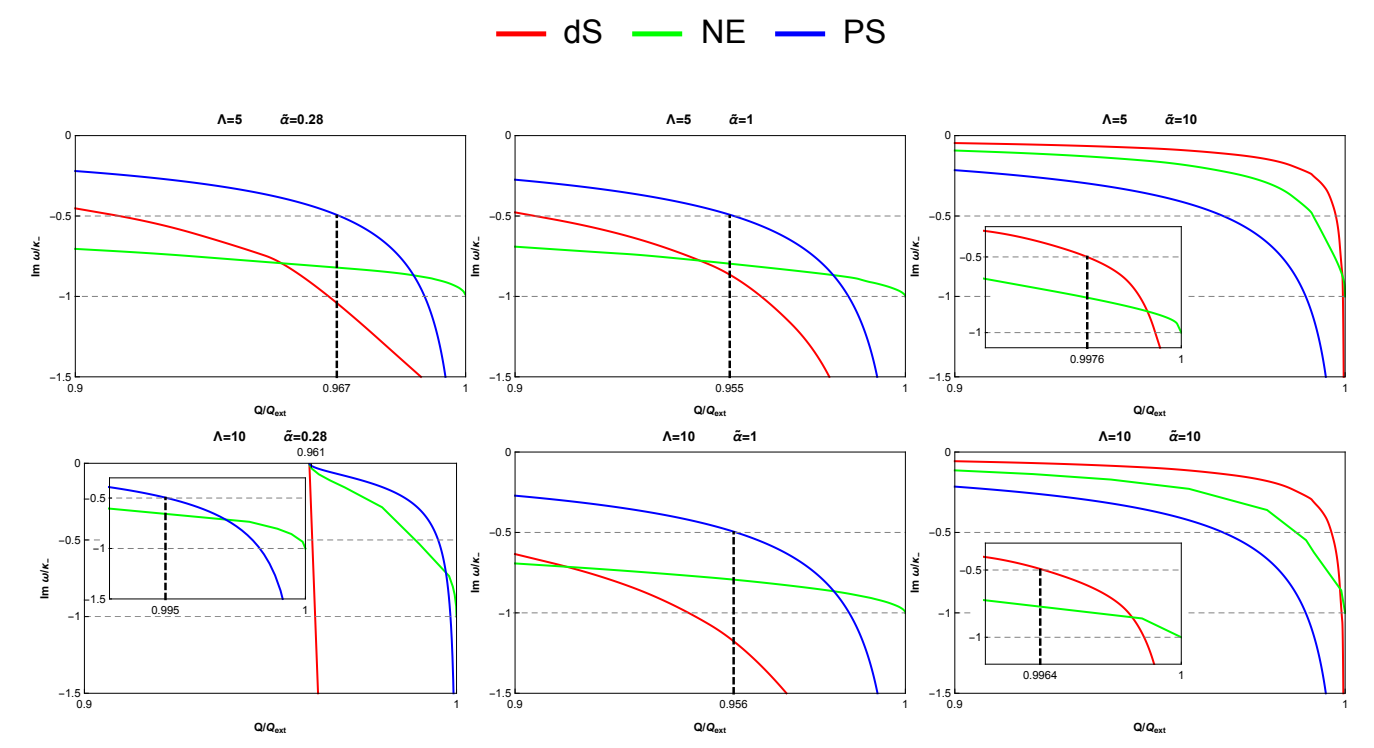

(a) Dominant modes of three families for various values of $\widetilde{\alpha}$ and $\Lambda$ with varying $Q / Q_{\text {ext }}$.

The SCC violation range of $Q / Q_{\text {ext }}$ first expands and then shrinks as $\widetilde{\alpha}$ increases.
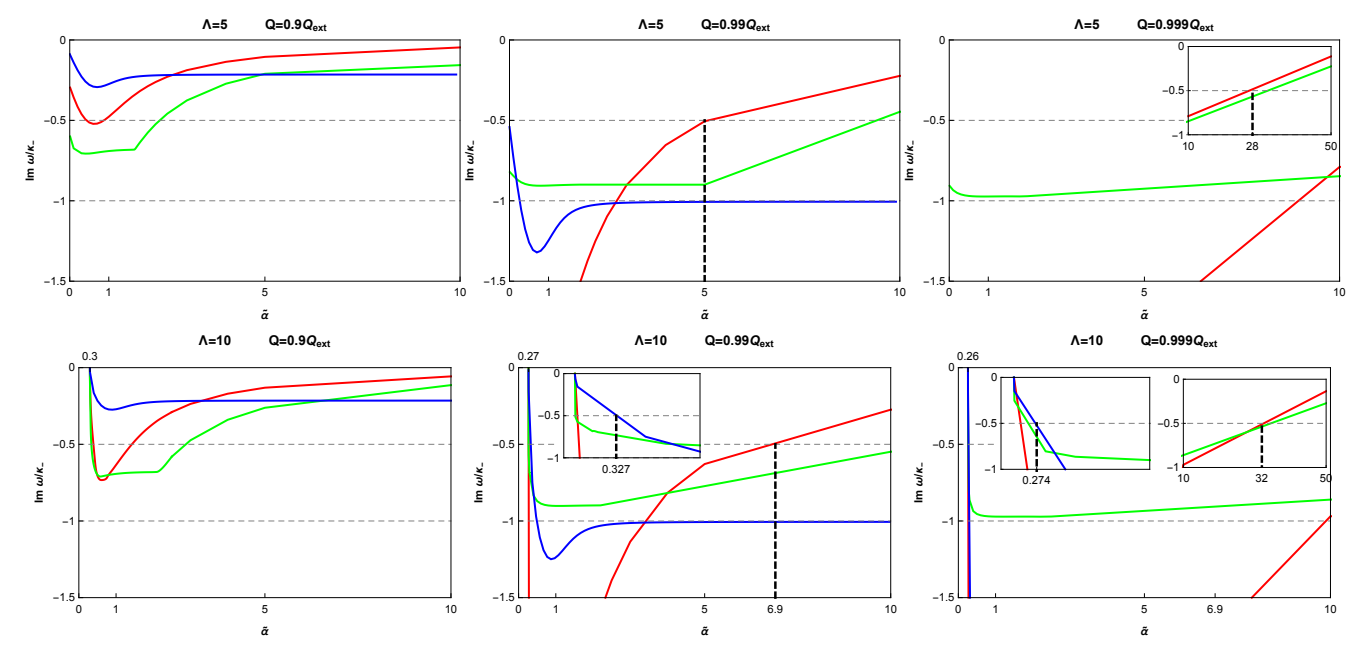

(b) Dominant modes of three families for various values of $Q / Q_{\text {ext }}$ and $\Lambda$ with varying $\widetilde{\alpha}$.

SCC is restored when $\widetilde{\alpha}$ is large enough.

FIG. 5: Dominant modes of three families for a neutral massless scalar field in a 6-dimensional EMGBdS black hole. The vertical thick dashed lines designate the points where $\beta=1 / 2$.

5. It shows that the ranges of $Q / Q_{\text {ext }}$, in which $\mathrm{SCC}$ is violated, first increase and then decrease with increasing $\widetilde{\alpha}$. Furthermore, we plot $\operatorname{Im}(\omega) / \kappa_{-}$of three families' dominant modes against $\widetilde{\alpha}$ with fixed $Q / Q_{\text {ext }}$ in Fig. 5b. When $\Lambda=5<\Lambda_{\mathrm{c}}$, one has $\widetilde{\alpha} \geq 0$. However when $\Lambda=10>\Lambda_{\mathrm{c}}$, there exists a positive lower bound on $\widetilde{\alpha}$, which can be observed in the 
lower row of Fig. 5b. Since the lower bound corresponds to the Nariai limit, SCC is always valid close to the lower bound. Fig. $5 \mathrm{~b}$ shows that, as $\widetilde{\alpha}$ increases from its minimum value, $\operatorname{Im}(\omega) / \kappa_{-}$of the three families' dominant modes all first decrease, and then the $\mathrm{dS}$ and $\mathrm{NE}$ modes increase to zero while the PS mode increases to some negative constant. Therefore SCC is always valid as long as $\widetilde{\alpha}$ is large enough. Moreover, the SCC violation regions in Fig. 5b, expand with increasing $Q / Q_{\text {ext }}$, which indicates that SCC tends to be violated for a black hole closer to extremality.

The density plots of $\beta$ for 6 -dimensional EMGBdS black holes with $\Lambda=5$ and $\Lambda=10$ are displayed in Fig. 6, where SCC is violated in the regions between the extremal limit (dashed orange lines) and the threshold $\beta=1 / 2$ (solid black lines). For a near-extremal black hole with $Q / Q_{\text {ext }}=0.99$ and $\Lambda=5<\Lambda_{\mathrm{c}}$ (the red line), the upper row of Fig. 6 shows that SCC is violated for small $\widetilde{\alpha}$, but can be restored when $\widetilde{\alpha} \gtrsim 5.2$. When $\Lambda=10>\Lambda_{\mathrm{c}}$, the lower left panel of Fig. 6 highlights the parameter space around the lower bound on $\widetilde{\alpha}$ (point A), which shows that SCC is always valid when black holes approach the Nariai limit. We present $\operatorname{Im}(\omega) / \kappa_{-}$of the dominant NE modes for various near-extremal values of $Q / Q_{\text {ext }}$ with $\Lambda=1$ and $\widetilde{\alpha}=1$ in the table of Fig. 6. Like the RNdS case, we observe that $\beta$ approaches 1 from below in the extremal limit, and hence the $C^{2}$ version of SCC is respected in the $d=6$ case.

\section{DISCUSSION AND CONCLUSION}

In this paper, we investigated the validity of SCC for a linear neutral massless scalar perturbation in 5- and 6-dimensional EMGBdS black holes. In Sec. II] we obtained the allowed parameter regions where a EMGBdS black hole can possess the Cauchy horizon for various $\Lambda$. After the method to calculate QNMs was discussed in Sec. III, the numerical results were presented in Sec. IV]

For the EMGBdS black holes in the allowed region, there are two limits, namely the extremal limit and the Nariai limit. In the extremal limit, we numerically found that $\beta \rightarrow 1$, and hence SCC is always violated. However in the Nariai limit, we showed that $\beta \rightarrow 0$, and hence SCC is always valid. When $\Lambda>\Lambda_{\mathrm{c}}$, the GB parameter $\widetilde{\alpha}$ was found to have a positive lower bound, which corresponds to the Nariai black hole. So SCC is respected near the lower bound on $\widetilde{\alpha}$. On the other hand, there is an upper bound on $\widetilde{\alpha}$ in the $d=5$ case while no 

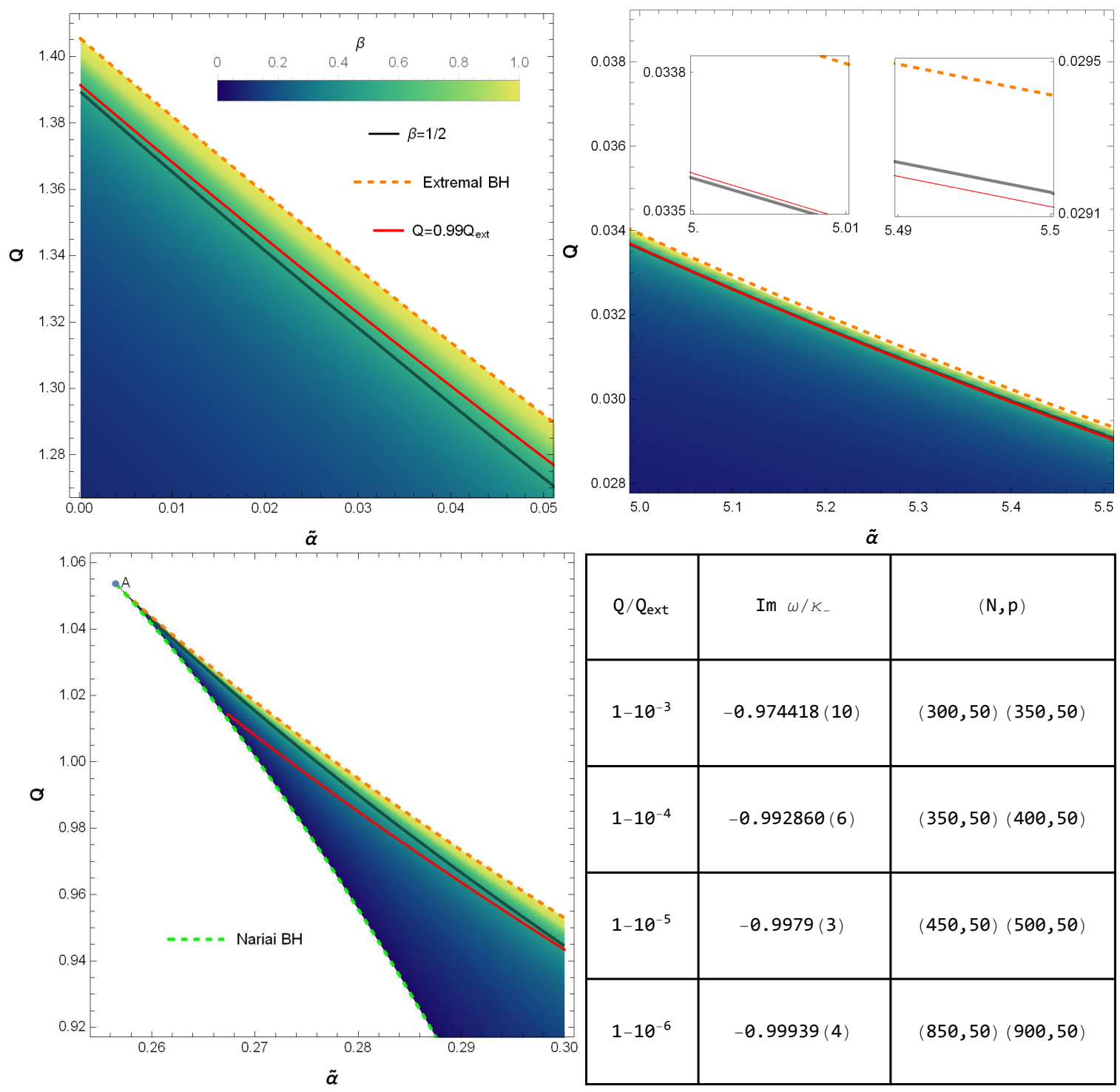

\begin{tabular}{|c|c|c|}
\hline $\mathrm{Q} / Q_{\text {ext }}$ & $\mathrm{Im} \omega / \mathcal{K}_{-}$ & $(\mathrm{N}, \mathrm{p})$ \\
\hline $1-10^{-3}$ & $-0.974418(10)$ & $(300,50)(350,50)$ \\
\hline $1-10^{-4}$ & $-0.992860(6)$ & $(350,50)(400,50)$ \\
\hline $1-10^{-5}$ & $-0.9979(3)$ & $(450,50)(500,50)$ \\
\hline $1-10^{-6}$ & $-0.99939(4)$ & $(850,50)(900,50)$ \\
\hline
\end{tabular}

FIG. 6: Upper Row: Density plots of $\beta$ for a neutral massless scalar field in a 6-dimensional EMGBdS black hole with $\Lambda=5$. The SCC violation regions are between $\beta=1 / 2$ (solid black lines) and the extremal limit (orange dashed lines). The insets shows that the $Q / Q_{\text {ext }}=0.99$ line (red line) exits the SCC violation region around $\widetilde{\alpha} \gtrsim 5.2$. Lower Left Panel: Density plots of $\beta$ in a 6-dimensional EMGBdS black hole with $\Lambda=10>\Lambda_{\mathrm{c}}$ around the minimum value of $\widetilde{\alpha}$. SCC is always saved close enough to the Nariai limit (dashed green lines). Lower Right Panel: A table of $\operatorname{Im}(\omega) / \kappa_{-}$of the dominant NE modes for 6-dimensional EMGBdS black holes with $\Lambda=1$ and $\widetilde{\alpha}=1$, which suggests that $\beta<1$ in the highly near-extremal case and $\beta \rightarrow 1$ in the extremal limit. 


\begin{tabular}{|l|c|c|}
\hline & SCC violation range of $Q / Q_{\text {ext }}$ with fixed $\widetilde{\alpha}$ & Varying $\widetilde{\alpha}$ with fixed $Q / Q_{\text {ext }}$ \\
\hline$d=5$ & increases as $\widetilde{\alpha}$ increases & SCC is violated near the maximum value of $\widetilde{\alpha}$ \\
\hline$d=6$ & first increases and then decreases as $\widetilde{\alpha}$ increases & SCC is restored when $\widetilde{\alpha}$ is large enough \\
\hline
\end{tabular}

TABLE I: The dependence of the validity of SCC on the GB parameter $\widetilde{\alpha}$.

upper bound is imposed on $\widetilde{\alpha}$ in the $d=6$ case. Our numerical results displayed that SCC tends to be violated/saved when $\widetilde{\alpha}$ is large enough in the $d=5 / d=6$ case, which implies the GB term inclines to worsen/alleviate the violation of SCC in the $d=5 / d=6$ case. We summarize the results about how the validity of SCC depends on $\widetilde{\alpha}$ in Table I, which indicates that the GB term plays a different role in the validity of SCC for a 5-dimensional EMGBdS black hole than it does for a 6-dimensional one.

We also checked the validity of the $C^{2}$ version of SCC for near-extremal EMGBdS black holes. In the $d=5$ case, we found that in some parameter region, $\beta$ is allowed to exceed unity, which implies that the scalar is in $C^{1}$ extension on the Cauchy horizon, and hence SCC is violated in the $C^{2}$ version. Such violation leads to the existence of solutions with bounded Ricci curvature, corresponding to a much more severe failure of determinism in General Relativity. However, the violation of SCC in the $C^{2}$ version has not been observed in the $d=6$ case. Furthermore, we numerically found that $\beta \rightarrow 1$ in the extremal limit for both $d=5$ and $d=6$. To our knowledge, the results of charged black holes in EinsteinMaxwell theory [23, 37], Einstein-Born-Infeld theory [42], Einstein-Logarithmic theory [43] and Horndeski theory [44] all suggest that the dominant mode of NE family approaches 1 in the extremal limit. It is known that such mode has been described analytically in asymptotically flat spacetime [36, 66, 67]. The reason why $\beta \rightarrow 1$ in the extremal limit may relate to the fact that the extremal black holes share the same near horizon topology, namely $A d S_{2} \times S^{d-2}$, leading to an enhanced spacetime symmetry [68 72$]$.

For EMGBdS black holes with $d>6$, we expect that the result might be similar to the $d=6$ case since their allowed regions are alike. In this paper, we only considered the scalar field perturbation on the fixed EMGBdS black hole background in the probe limit without taking into account the backreaction of the scalar field on the black hole spacetime. So our results on stability actually refer to the scalar field rather than the EMGBdS black hole spacetime. In the future studies, it is very interesting to check the validity of SCC and 
discuss its dependence on the dimension $d$ in a full backreaction way .

Note: Just before this paper was submitted to arXiv, a relevant preprint [73] appeared, which investigated SCC in higher curvature gravity. In [73], it was found that the violation of SCC becomes worse as $\widetilde{\alpha}$ increases in the small $\widetilde{\alpha}$ regime for both $d=5$ and $d=6$, which is in agreement with our results. However, we carry out the analysis in a more through way with a broader survey of the parameter space and show that the behavior of SCC in the $d=5$ and $d=6$ cases is quite different, which was not observed in [73]. Specifically, in the large $\widetilde{\alpha}$ regime in the $d=6$ case, we display that the GB term tends to alleviate the violation of SCC.

Acknowledgements. We thank Guangzhou Guo and Shuxuan Ying for their helpful discussions and suggestions. This work is supported in part by NSFC (Grant No. 11005016, 11875196 and 11375121).

[1] R. Penrose. Gravitational collapse: The role of general relativity. Riv. Nuovo Cim., 1:252-276, 1969. [Gen. Rel. Grav.34,1141(2002)].

[2] S. W. Hawking and R. Penrose. The Singularities of gravitational collapse and cosmology. Proc. Roy. Soc. Lond., A314:529-548, 1970. doi:10.1098/rspa.1970.0021.

[3] Roger Penrose. Gravitational collapse and space-time singularities. Phys. Rev. Lett., 14:57-59, 1965. doi:10.1103/PhysRevLett.14.57.

[4] Jonathan Luk and Sung-Jin Oh. Strong cosmic censorship in spherical symmetry for twoended asymptotically flat initial data I. The interior of the black hole region. 2017. arXiv: 1702.05715.

[5] Jonathan Luk and Sung-Jin Oh. Strong cosmic censorship in spherical symmetry for twoended asymptotically flat initial data II. The exterior of the black hole region. 2017. arXiv: 1702.05716 .

[6] J.M. McNamara. Behaviour of scalar perturbations of a Reissner-Nordstroem black hole inside the event horizon. Proceedings of the Royal Society of London Series A, Mathematical and Physical Sciences, 364(1716):121-134, 1978. doi:https://doi.org/10.1098/rspa.1978.0191.

[7] Mihalis Dafermos. The Interior of charged black holes and the problem of uniqueness in general 
relativity. Commun. Pure Appl. Math., 58:0445-0504, 2005. arXiv:gr-qc/0307013.

[8] Anne T. Franzen. Boundedness of Massless Scalar Waves on Reissner-Nordström Interior Backgrounds. Commun. Math. Phys., 343(2):601-650, 2016. arXiv:1407.7093, doi:10.1007/ s00220-015-2440-7.

[9] Eric Poisson and W. Israel. Internal structure of black holes. Phys. Rev., D41:1796-1809, 1990. doi:10.1103/PhysRevD.41.1796

[10] Amos Ori. Inner structure of a charged black hole: An exact mass-inflation solution. Phys. Rev. Lett., 67:789-792, 1991. doi:10.1103/PhysRevLett.67.789.

[11] Demetrios Christodoulou. The Formation of Black Holes in General Relativity. In On recent developments in theoretical and experimental general relativity, astrophysics and relativistic field theories. Proceedings, 12th Marcel Grossmann Meeting on General Relativity, Paris, France, July 12-18, 2009. Vol. 1-3, pages 24-34, 2008. arXiv:0805.3880, doi: 10.1142/9789814374552_0002.

[12] Chris M. Chambers. The Cauchy horizon in black hole de sitter space-times. Annals Israel Phys. Soc., 13:33, 1997. [,33(1997)]. arXiv:gr-qc/9709025.

[13] Shahar Hod and Tsvi Piran. Mass inflation in dynamical gravitational collapse of a charged scalar field. Phys. Rev. Lett., 81:1554-1557, 1998. arXiv:gr-qc/9803004, doi:10.1103/ PhysRevLett.81.1554.

[14] Patrick R. Brady and John D. Smith. Black hole singularities: A Numerical approach. Phys. Rev. Lett., 75:1256-1259, 1995. arXiv:gr-qc/9506067, doi:10.1103/PhysRevLett.75.1256.

[15] Richard H. Price. Nonspherical perturbations of relativistic gravitational collapse. 1. Scalar and gravitational perturbations. Phys. Rev., D5:2419-2438, 1972. doi:10.1103/PhysRevD.5.2419.

[16] Mihalis Dafermos. Black holes without spacelike singularities. Commun. Math. Phys., 332:729 757, 2014. arXiv:1201.1797, doi:10.1007/s00220-014-2063-4.

[17] Mihalis Dafermos, Igor Rodnianski, and Yakov Shlapentokh-Rothman. Decay for solutions of the wave equation on Kerr exterior spacetimes III: The full subextremal case $|a|<$ M. 2014. arXiv:1402.7034

[18] Yannis Angelopoulos, Stefanos Aretakis, and Dejan Gajic. Late-time asymptotics for the wave equation on spherically symmetric, stationary spacetimes. Adv. Math., 323:529-621, 2018. arXiv:1612.01566, doi:10.1016/j.aim.2017.10.027.

[19] João L. Costa, Pedro M. Girão, José Natário, and Jorge Drumond Silva. On the global 
uniqueness for the Einstein-Maxwell-scalar field system with a cosmological constant. Part 3: Mass inflation and extendibility of the solutions. 2014. arXiv:1406.7261, doi:10.1007/ s40818-017-0028-6.

[20] João L. Costa, Pedro M. Girão, José Natário, and Jorge Drumond Silva. On the global uniqueness for the Einstein-Maxwell-scalar field system with a cosmological constant: I. Well posedness and breakdown criterion. Class. Quant. Grav., 32(1):015017, 2015. arXiv:1406. 7245, doi:10.1088/0264-9381/32/1/015017

[21] João L. Costa, Pedro M. Girão, José Natário, and Jorge Drumond Silva. On the global uniqueness for the Einstein-Maxwell-scalar field system with a cosmological constant. Commun. Math. Phys., 339(3):903-947, 2015. arXiv:1406.7253, doi:10.1007/s00220-015-2433-6.

[22] Peter Hintz and András Vasy. Analysis of linear waves near the Cauchy horizon of cosmological black holes. J. Math. Phys., 58(8):081509, 2017. arXiv:1512.08004, doi:10.1063/1.4996575.

[23] Vitor Cardoso, João L. Costa, Kyriakos Destounis, Peter Hintz, and Aron Jansen. Quasinormal modes and Strong Cosmic Censorship. Phys. Rev. Lett., 120(3):031103, 2018. arXiv:1711. 10502, doi:10.1103/PhysRevLett.120.031103.

[24] Christoph Kehle and Yakov Shlapentokh-Rothman. A scattering theory for linear waves on the interior of Reissner-Nordström black holes. Annales Henri Poincare, 20(5):1583-1650, 2019. arXiv:1804.05438, doi:10.1007/s00023-019-00760-z.

[25] Oscar J. C. Dias, Harvey S. Reall, and Jorge E. Santos. Strong cosmic censorship: taking the rough with the smooth. JHEP, 10:001, 2018. arXiv:1808.02895, doi:10.1007/JHEP10(2018) 001 .

[26] Vitor Cardoso, Joao L. Costa, Kyriakos Destounis, Peter Hintz, and Aron Jansen. Strong cosmic censorship in charged black-hole spacetimes: still subtle. Phys. Rev., D98(10):104007, 2018. arXiv:1808.03631, doi:10.1103/PhysRevD.98.104007.

[27] Yuyu Mo, Yu Tian, Bin Wang, Hongbao Zhang, and Zhen Zhong. Strong cosmic censorship for the massless charged scalar field in the Reissner-Nordstrom-de Sitter spacetime. Phys. Rev., D98(12):124025, 2018. arXiv:1808.03635, doi:10.1103/PhysRevD.98.124025.

[28] Oscar J. C. Dias, Harvey S. Reall, and Jorge E. Santos. Strong cosmic censorship for charged de Sitter black holes with a charged scalar field. Class. Quant. Grav., 36(4):045005, 2019. arXiv:1808.04832, doi:10.1088/1361-6382/aafcf2,

[29] Shahar Hod. Strong cosmic censorship in charged black-hole spacetimes: As strong as ever. 
Nucl. Phys., B941:636-645, 2019. arXiv:1801.07261, doi:10.1016/j.nuclphysb.2019.03. 003.

[30] Mihalis Dafermos and Yakov Shlapentokh-Rothman. Rough initial data and the strength of the blue-shift instability on cosmological black holes with $\Lambda>0$. Class. Quant. Grav., 35(19):195010, 2018. arXiv:1805.08764, doi:10.1088/1361-6382/aadbcf.

[31] Yongwan Gim and Bogeun Gwak. Charged Particle and Strong Cosmic Censorship in ReissnerNordström-de Sitter Black Holes. 2019. arXiv:1901.11214.

[32] Boxuan Ge, Jie Jiang, Bin Wang, Hongbao Zhang, and Zhen Zhong. Strong cosmic censorship for the massless Dirac field in the Reissner-Nordstrom-de Sitter spacetime. JHEP, 01:123, 2019. arXiv:1810.12128, doi:10.1007/JHEP01(2019) 123

[33] Kyriakos Destounis. Charged Fermions and Strong Cosmic Censorship. Phys. Lett., B795:211219, 2019. arXiv:1811.10629, doi:10.1016/j.physletb.2019.06.015.

[34] Hongbao Zhang and Zhen Zhong. Strong cosmic censorship in de Sitter space: As strong as ever. 2019. arXiv:1910.01610.

[35] Mostafizur Rahman. On the validity of Strong Cosmic Censorship Conjecture in presence of Dirac fields. 2019. arXiv:1905.06675.

[36] Mostafizur Rahman, Sumanta Chakraborty, Soumitra SenGupta, and Anjan A. Sen. Fate of Strong Cosmic Censorship Conjecture in Presence of Higher Spacetime Dimensions. JHEP, 03:178, 2019. arXiv:1811.08538, doi:10.1007/JHEP03(2019)178.

[37] Hang Liu, Ziyu Tang, Kyriakos Destounis, Bin Wang, Eleftherios Papantonopoulos, and Hongbao Zhang. Strong Cosmic Censorship in higher-dimensional Reissner-Nordstrom-de Sitter spacetime. JHEP, 03:187, 2019. arXiv:1902.01865, doi:10.1007/JHEP03(2019)187

[38] Xiaoyi Liu, Stijn Van Vooren, Hongbao Zhang, and Zhen Zhong. Strong cosmic censorship for the Dirac field in the higher dimensional Reissner-Nordstrom-de Sitter black hole. JHEP, 10:186, 2019. arXiv:1909.07904, doi:10.1007/JHEP10(2019)186.

[39] Raimon Luna, Miguel Zilhao, Vitor Cardoso, Joao L. Costa, and Jose Natario. Strong Cosmic Censorship: the nonlinear story. Phys. Rev., D99(6):064014, 2019. arXiv:1810.00886, doi: 10.1103/PhysRevD.99.064014.

[40] Bogeun Gwak. Strong Cosmic Censorship under Quasinormal Modes of Non-Minimally Coupled Massive Scalar Field. 2018. arXiv:1812.04923

[41] Oscar J. C. Dias, Felicity C. Eperon, Harvey S. Reall, and Jorge E. Santos. Strong cosmic 
censorship in de Sitter space. Phys. Rev., D97(10):104060, 2018. arXiv:1801.09694, doi: 10.1103/PhysRevD.97.104060.

[42] Qingyu Gan, Guangzhou Guo, Peng Wang, and Houwen Wu. Strong Cosmic Censorship for a Scalar Field in a Born-Infeld-de Sitter Black Hole. 2019. arXiv:1907.04466.

[43] Yiqian Chen, Qingyu Gan, and Guangzhou Guo. Strong Cosmic Censorship for a Scalar Field in a Logarithmic-de Sitter Black Hole. 2019. arXiv:1911.06628.

[44] Kyriakos Destounis, Rodrigo D. B. Fontana, Filipe C. Mena, and Eleftherios Papantonopoulos. Strong Cosmic Censorship in Horndeski Theory. 2019. arXiv:1908.09842.

[45] David G. Boulware and Stanley Deser. String Generated Gravity Models. Phys. Rev. Lett., 55:2656, 1985. doi:10.1103/PhysRevLett.55.2656.

[46] D. L. Wiltshire. Spherically Symmetric Solutions of Einstein-maxwell Theory With a GaussBonnet Term. Phys. Lett., 169B:36-40, 1986. doi:10.1016/0370-2693(86)90681-7.

[47] Rong-Gen Cai. Gauss-Bonnet black holes in AdS spaces. Phys. Rev., D65:084014, 2002. arXiv:hep-th/0109133, doi:10.1103/PhysRevD.65.084014.

[48] Rong-Gen Cai and Qi Guo. Gauss-Bonnet black holes in dS spaces. Phys. Rev., D69:104025, 2004. arXiv:hep-th/0311020, doi:10.1103/PhysRevD.69.104025.

[49] Roman Konoplya. Quasinormal modes of the charged black hole in Gauss-Bonnet gravity. Phys. Rev., D71:024038, 2005. arXiv:hep-th/0410057, doi:10.1103/PhysRevD.71.024038.

[50] Takashi Torii and Hideki Maeda. Spacetime structure of static solutions in Gauss-Bonnet gravity: Charged case. Phys. Rev., D72:064007, 2005. arXiv:hep-th/0504141, doi:10.1103/ PhysRevD.72.064007.

[51] Decheng Zou, Zhanying Yang, Ruihong Yue, and Peng Li. Thermodynamics of Gauss-BonnetBorn-Infeld black holes in AdS space. Mod. Phys. Lett., A26:515-529, 2011. arXiv:1011.3184, doi:10.1142/S0217732311034724.

[52] Peng Wang, Haitang Yang, and Shuxuan Ying. Thermodynamics and Phase Transition of a Gauss-Bonnet Black Hole in a Cavity. 2019. arXiv:1909.01275.

[53] Xiao-Xiong Zeng, Xin-Yun Hu, and Ke-Jian He. Weak cosmic censorship conjecture with pressure and volume in the Gauss-Bonnet AdS black hole. 2019. arXiv:1905.07750.

[54] Marc Thibeault, Claudio Simeone, and Ernesto F. Eiroa. Thin-shell wormholes in EinsteinMaxwell theory with a Gauss-Bonnet term. Gen. Rel. Grav., 38:1593-1608, 2006. arXiv: gr-qc/0512029, doi:10.1007/s10714-006-0324-z 
[55] Atsushi Higuchi. Symmetric Tensor Spherical Harmonics on the $N$ Sphere and Their Application to the De Sitter Group $\mathrm{SO}(N, 1)$. J. Math. Phys., 28:1553, 1987. [Erratum: J. Math. Phys.43,6385(2002)]. doi:10.1063/1.527513.

[56] Octavio Fierro, Nicolas Grandi, and Julio Oliva. Superradiance of charged black holes in Einstein-Gauss-Bonnet gravity. Class. Quant. Grav., 35(10):105007, 2018. arXiv:1708.06037, doi:10.1088/1361-6382/aab3f6.

[57] Emanuele Berti, Vitor Cardoso, and Andrei O. Starinets. Quasinormal modes of black holes and black branes. Class. Quant. Grav., 26:163001, 2009. arXiv:0905.2975, doi:10.1088/ $0264-9381 / 26 / 16 / 163001$.

[58] R. A. Konoplya and A. Zhidenko. Quasinormal modes of black holes: From astrophysics to string theory. Rev. Mod. Phys., 83:793-836, 2011. arXiv:1102.4014, doi:10.1103/ RevModPhys .83.793.

[59] Aron Jansen. Overdamped modes in Schwarzschild-de Sitter and a Mathematica package for the numerical computation of quasinormal modes. Eur. Phys. J. Plus, 132(12):546, 2017. arXiv:1709.09178, doi:10.1140/epjp/i2017-11825-9.

[60] Aron Peter Jansen. Qnmspectral. URL: https://github.com/APJansen/QNMspectral.

[61] URL: https://centra.tecnico.ulisboa.pt/network/grit/files/ringdown/

[62] Akihiro Ishibashi and Hideo Kodama. Stability of higher dimensional Schwarzschild black holes. Prog. Theor. Phys., 110:901-919, 2003. arXiv:hep-th/0305185, doi:10.1143/PTP.110.901.

[63] E. Abdalla, R. A. Konoplya, and C. Molina. Scalar field evolution in Gauss-Bonnet black holes. Phys. Rev., D72:084006, 2005. arXiv:hep-th/0507100, doi:10.1103/PhysRevD.72.084006.

[64] R. A. Konoplya and A. Zhidenko. (In)stability of D-dimensional black holes in Gauss-Bonnet theory. Phys. Rev., D77:104004, 2008. arXiv:0802.0267, doi:10.1103/PhysRevD.77.104004.

[65] Clarissa-Marie Claudel, K. S. Virbhadra, and G. F. R. Ellis. The Geometry of photon surfaces. J. Math. Phys., 42:818-838, 2001. arXiv:gr-qc/0005050, doi:10.1063/1.1308507.

[66] Chiang-Mei Chen, Sang Pyo Kim, I-Chieh Lin, Jia-Rui Sun, and Ming-Fan Wu. Spontaneous Pair Production in Reissner-Nordstrom Black Holes. Phys. Rev., D85:124041, 2012. arXiv: 1202.3224, doi:10.1103/PhysRevD.85.124041.

[67] Yong-Wan Kim, Yun Soo Myung, and Young-Jai Park. Quasinormal modes and hidden conformal symmetry in the Reissner-Nordstróm black hole. Eur. Phys. J., C73:2440, 2013. arXiv:1205.3701, doi:10.1140/epjc/s10052-013-2440-8. 
[68] Vijay Balasubramanian and Finn Larsen. Near horizon geometry and black holes in fourdimensions. Nucl. Phys., B528:229-237, 1998. arXiv:hep-th/9802198, doi:10.1016/ S0550-3213(98)00334-4.

[69] Mirjam Cvetic and Finn Larsen. Near horizon geometry of rotating black holes in fivedimensions. Nucl. Phys., B531:239-255, 1998. arXiv:hep-th/9805097, doi:10.1016/ S0550-3213(98)00604-X.

[70] Jin-Ho Cho and Soonkeon Nam. Non-supersymmetric attractor with the cosmological constant. JHEP, 07:011, 2007. arXiv:0705.2892, doi:10.1088/1126-6708/2007/07/011

[71] Hari K. Kunduri, James Lucietti, and Harvey S. Reall. Near-horizon symmetries of extremal black holes. Class. Quant. Grav., 24:4169-4190, 2007. arXiv:0705.4214, doi: $10.1088 / 0264-9381 / 24 / 16 / 012$.

[72] Hari K. Kunduri and James Lucietti. A Classification of near-horizon geometries of extremal vacuum black holes. J. Math. Phys., 50:082502, 2009. arXiv:0806.2051, doi:10.1063/1. 3190480 .

[73] Akash K. Mishra and Sumanta Chakraborty. Strong Cosmic Censorship in higher curvature gravity. 2019. arXiv:1911.09855. 